\title{
Communication
}

\section{In Crystallo Capture of a Michaelis Complex and Product-binding Modes of a Bacterial Phosphotriesterase}

\author{
Colin J. Jackson†, Jee-Loon Foo†, Hye-Kyung Kim, Paul D. Carr, \\ Jian-Wei Liu, Geoffrey Salem and David L. Ollis*
}

Research School of Chemistry, Australian National University, Australian Capital Territory 0200, Australia

Received 5 September 2007; received in revised form 19 October 2007; accepted 24 October 2007 Available online 1 November 2007

\begin{abstract}
The mechanism by which the binuclear metallophosphotriesterases (PTEs, E.C. 3.1.8.1) catalyse substrate hydrolysis has been extensively studied. The $\mu$-hydroxo bridge between the metal ions has been proposed to be the initiating nucleophile in the hydrolytic reaction. In contrast, analysis of some biomimetic systems has indicated that $\mu$-hydroxo bridges are often not themselves nucleophiles, but act as general bases for freely exchangeable nucleophilic water molecules. Herein, we present crystallographic analyses of a bacterial PTE from Agrobacterium radiobacter, OpdA, capturing the enzyme-substrate complex during hydrolysis. This model of the Michaelis complex suggests the alignment of the substrate will favour attack from a solvent molecule terminally coordinated to the $\alpha$-metal ion. The bridging of both metal ions by the product, without disruption of the $\mu$-hydroxo bridge, is also consistent with nucleophilic attack occurring from the terminal position. When phosphodiesters are soaked into crystals of OpdA, they coordinate bidentately to the $\beta$-metal ion, displacing the $\mu$-hydroxo bridge. Thus, alternative product-binding modes exist for the PTEs, and it is the bridging mode that appears to result from phosphotriester hydrolysis. Kinetic analysis of the PTE and promiscuous phosphodiesterase activities confirms that the presence of a $\mu$-hydroxo bridge during phosphotriester hydrolysis is correlated with a lower $\mathrm{p} K_{\mathrm{a}}$ for the nucleophile, consistent with a general base function during catalysis.
\end{abstract}

(C) 2007 Elsevier Ltd. All rights reserved.

Keywords: binuclear metal centre; phosphotriesterase; general base; $\mu$ hydroxo bridge; Michaelis complex ligands. ${ }^{3}$ OpdA natively contains a heterobinuclear $\mathrm{Fe}^{2+}-\mathrm{Zn}^{2+}$ active site, ${ }^{4}$ although PTEs are catalytically active with a wide variety of divalent metal ions. ${ }^{5}$ PTEs catalyse the hydrolysis of phosphotriesters via a single in-line displacement $\left(\mathrm{S}_{\mathrm{N}} 2\right)$ mechanism involving direct in-line attack from a metalcoordinated hydroxide molecule at the electrophilic phosphorus. ${ }^{6}$ The identity of the nucleophilic species has been proposed to be either a hydroxide molecule terminally coordinated to the $\alpha-$ metal $^{7}$ or the $\mu$-hydroxo bridge between the $\alpha$ - and $\beta$-metals. ${ }^{8}$ Efforts to rationalise the catalytic mechanism have met with mixed success. For instance, the observations that in-crystal substrate hydrolysis results in the product dually bound to both metal ions, without disruption of the $\mu$-hydroxo bridge, ${ }^{7}$ and 
enzyme kinetics showing the $\alpha$-metal ion affects the $\mathrm{p} K_{\mathrm{a}}$ of the nucleophile, ${ }^{8}$ support a water nucleophile terminally coordinated to the $\alpha$-metal ion. In contrast, the $\mathrm{p} K_{\mathrm{a}}$ of the nucleophilic species is closely correlated with the $\mathrm{p} K_{\mathrm{a}}$ of the $\mu$-hydroxo bridge, ${ }^{9}$ and only one ionizable group is responsible for the increase in the catalytic rate, ${ }^{10}$ supporting a $\mu$-hydroxo bridge nucleophile.

The understanding of binuclear metal centers in biology has been greatly advanced through the use of biomimetic models. ${ }^{11}$ It has been suggested from such studies that despite the low $\mathrm{p} K_{\mathrm{a}}$ values of $\mu$-hydroxo bridges, they are relatively poor nucleophiles and are often not well oriented for nucleophilic attack. ${ }^{12}$ More recent work has shown free coordination sites for a nucleophilic water near the $\mu$-hydroxo bridge significantly enhance the catalytic rate, and the $\mu$-hydroxo bridge can act as a general base, lowering the $p K_{a}$ of a terminally coordinated water molecule almost as efficiently as a metal ion. ${ }^{15,16}$ The recent construction of a functional model of the PTE active site demonstrated that reasonable catalysis is possible when the $\mu$-hydroxo bridge is replaced by a phenoxide group that could not act as a nucleophile, suggesting the $\mu$-hydroxo bridge is unlikely to be the nucleophilic species. ${ }^{13}$

\section{Substrate turnover in crystallo and distinct phosphodiester-binding modes}

In an effort to reconcile the different mechanistic models, a substrate with a poor leaving group, diethyl 4-methoxyphenyl phosphate (EPO), was synthesised on the basis that it would be turned over slowly enough to be observed in crystallo. An introductory scheme of the hydrolysis of the substrates used in this report is shown in Fig. 1. It is well documented that EPO is turned over very slowly by the PTEs $\left(2 \times 10^{5}\right.$-fold more slowly than the "fast" substrate paraoxon) and the rate-limiting step in its turnover is chemical hydrolysis. ${ }^{14}$ Our own measurements confirm the turnover is extremely slow $\left(9.8 \times 10^{-3} \mu \mathrm{mol} \mathrm{min}{ }^{-1} \mu \mathrm{mol}^{-1}\right.$ of OpdA) and indicate that only $\sim 5 \%$ of a $1 \mathrm{mM}$ EPO solution was degraded over $20 \mathrm{~h}$ by $5 \mu \mathrm{M}$ OpdA at pH 6.5.

EPO was soaked into a crystal of OpdA and data were collected on the same crystal after $30 \mathrm{~min}, 20 \mathrm{~h}$ and $450 \mathrm{~h}$ (Table 1). The structures collected after $30 \mathrm{~min}$ and $20 \mathrm{~h}$ were effectively identical but with less well defined electron density for the "30-min" structure. Figure 2a shows an omit electron density map of the 20-h time point, clearly showing EPO loosely (3.2 $\AA$ ) coordinated to the $\beta$-metal ion with $50 \%$ occupancy. This model of a Michaelis complex allows, for the first time, the geometry of the substrate to be observed prior to hydrolysis. There is hydrogen bonding between EPO and R254 and extensive $\pi-\pi$ interaction between four aromatic residues (Trp131, Phe132, Phe306 and Tyr309) in the leaving group channel and the phenyl group of the substrate (Fig. 3). After the substrate was included in the model and refined, improved phases revealed electron density that is consistent with the hydrolytic product diethyl phosphate (DEP) tightly bound in a bridging fashion to the active-site metals at low occupancy (15\%; Fig. 2b). This coordination mode would occur as a result of nucleophilic attack from an activated water molecule at the freely exchangeable terminal position at the $\alpha$-site and has previously been observed immediately after the in crystallo hydrolysis of dimethoate. ${ }^{7}$ After $450 \mathrm{~h}$, significant turnover had occurred with electron density present corresponding to DEP bound in a chelating binding mode at the $\beta$-metal ion and the leaving group in the leaving group channel, displacing the $\mu$-hydroxo bridge (Fig. $2 c$ and $d$ ). After these ligands were included in the model (50\% occupancy) and refined, residual electron density was visible, suggesting some substrate was still present, although the occupancy was less than half of that at the 20 -h time point (20\%). Thus, although the structures obtained after $20 \mathrm{~h}$ incubation suggest the reaction proceeds via attack at the electrophilic phosphorus by a terminally coordinated water/hydroxide nucleophile followed by formation of a bridging product, the product in the latter structure displaces the bridging hydroxide. In order to determine whether the product coordination in the 450 -h time point structure reflected product coordination directly resulting from nucleophilic attack from the $\mu$-hydroxo bridge or rebinding of the accumulated phosphodiester product, DEP was soaked into OpdA crystals for $24 \mathrm{~h}$. The result demonstrates that DEP displaces the $\mu$ hydroxo bridge upon binding (Fig. 2e), consistent with recent spectroscopic data. ${ }^{15}$ This chelating binding mode has also been observed in several phosphodiesterases. ${ }^{16,17}$

Having established that phosphotriesters bind terminally and produce a bridging product, whereas phosphodiesters displace the $\mu$-hydroxo bridge on binding, similar experiments were undertaken with

(a)<smiles>CCOP(=O)(O)OP(=O)(OCC)Oc1ccc(Oc2ccc([PH2+])cc2)cc1</smiles>

(b)

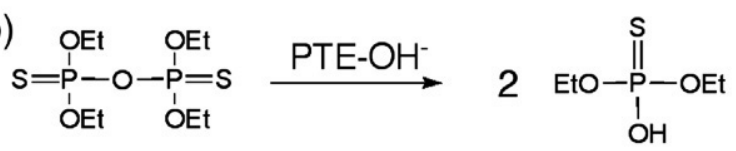

Fig. 1. Hydrolysis reactions of EPO (a) and TEDTPP (b) with PTE. 
Table 1. Data collection and refinement statistics for OpdA complexes

\begin{tabular}{|c|c|c|c|c|c|c|}
\hline & $\begin{array}{c}2 \mathrm{mM} \text { EPO, } \\
\text { crystal soaking } \\
(20 \mathrm{~h})\end{array}$ & $\begin{array}{c}2 \mathrm{mM} \text { EPO, } \\
\text { crystal soaking } \\
(450 \mathrm{~h})\end{array}$ & $\begin{array}{c}100 \mathrm{mM} \text { DEP, } \\
\text { crystal soaking } \\
(24 \mathrm{~h})\end{array}$ & $\begin{array}{c}40 \mu \mathrm{M} \text { TEDTPP, } \\
\text { cocrystallization } \\
\quad(>500 \mathrm{~h})\end{array}$ & $\begin{array}{c}40 \mu \mathrm{M} \text { TEDTPP, } \\
\text { crystal soaking } \\
(24 \mathrm{~h})\end{array}$ & $\begin{array}{c}10 \text { mM DETP, } \\
\text { crystal soaking } \\
(24 \mathrm{~h})\end{array}$ \\
\hline PDB code & $2 \mathrm{R} 1 \mathrm{~N}$ & 2R1M & 2R1K & 2R1P & $2 \mathrm{R} 1 \mathrm{O}$ & 2R1L \\
\hline \multicolumn{7}{|l|}{ Data collection } \\
\hline Cell dimensions $(a, c)(\AA)$ & $108.70,62.65$ & $109.10,61.88$ & $109.48,62.61$ & $108.88,62.84$ & $108.92,62.65$ & $108.92,62.65$ \\
\hline \multirow[t]{2}{*}{ Resolution $(\AA)^{\mathrm{a}}$} & $20-1.70$ & $20-2.50$ & $40-1.90$ & $20-1.80$ & $20-1.80$ & 20-1.95 \\
\hline & $(1.81-1.70)$ & $(2.66-2.50)$ & $(1.97-1.90)$ & $(1.91-1.80)$ & $(1.91-1.80)$ & $(2.07-1.95)$ \\
\hline$R_{\text {symm }}{ }^{\mathrm{b}}(\%)$ & $5.4(29.1)$ & $11.0(35.2)$ & $5.9(26.2)$ & $5.5(25.9)$ & $5.4(20.9)$ & $7.1(29.7)$ \\
\hline$I / \mathrm{ó}(I)$ & $22.6(2.0)$ & $11.3(2.1)$ & $55.1(5.8)$ & $25.2(4.3)$ & $45.1(4.9)$ & $19.7(3.6)$ \\
\hline Completeness (\%) & $95.2(73.2)$ & $96.0(82.5)$ & $96.4(77.0)$ & $95.8(94.2)$ & $98.9(93.8)$ & $99.3(96.3)$ \\
\hline Redundancy & $4.0(1.8)$ & $3.2(2.4)$ & $9.0(6.0)$ & $5.3(5.0)$ & $8.2(4.9)$ & $4.1(3.5)$ \\
\hline \multicolumn{7}{|l|}{ Refinement } \\
\hline \multirow[t]{2}{*}{ Resolution $(\AA)^{\mathrm{a}}$} & $18-1.70$ & $20-2.50$ & $25-2.10$ & $20-1.80$ & $20-1.80$ & 20-1.95 \\
\hline & $(1.74-1.70)$ & $(2.56-2.50)$ & $(2.15-2.10)$ & $(1.85-1.80)$ & $(1.85-1.80)$ & $(2.0-1.95)$ \\
\hline No. of reflections & 42,685 (1993) & $13,620(747)$ & 24,157 (1739) & $36,530(2612)$ & $37,486(2496)$ & 29,774 (1991) \\
\hline \multirow[t]{2}{*}{$R_{\text {work }} / R_{\text {free }}{ }^{c}(\%)$} & $16.5 / 19.0$ & $15.5 / 22.5$ & $15.3 / 20.2$ & $16.2 / 19.5$ & $17.2 / 18.8$ & $15.4 / 19.7$ \\
\hline & $(30.0 / 36.4)$ & $(20.9 / 33.2)$ & $(20.0 / 30.2)$ & $(21.1 / 24.0)$ & $(23.9 / 26.3)$ & $(18.0 / 27.9)$ \\
\hline \multicolumn{7}{|l|}{ No. of atoms } \\
\hline Protein & 2527 & 2504 & 2536 & 2506 & 2504 & 2511 \\
\hline Water & 317 & 153 & 290 & 288 & 287 & 272 \\
\hline $\mathrm{M}^{2+}$ ions & 2 & 2 & 2 & 2 & 2 & 2 \\
\hline Ligand & 2 & 2 & 1 & 1 & 1 & 1 \\
\hline Ethylene glycol & 0 & 1 & 1 & 1 & 1 & 1 \\
\hline \multicolumn{7}{|l|}{$B$-factor } \\
\hline Protein & 17.0 & 31.9 & 28.1 & 17.4 & 23.1 & 19.4 \\
\hline $\mathrm{M}^{2+}$ ions & 14.3 & 30.2 & 25.5 & 13.7 & 17.3 & 14.2 \\
\hline Ligands (primary) & 16.3 & 32.6 & 29.0 & 19.9 & 21.6 & 16.0 \\
\hline Ligands (secondary) & 11.7 & 27.0 & - & - & - & - \\
\hline Water & 28.9 & 35.8 & 39.5 & 28.7 & 35.5 & 28.7 \\
\hline \multicolumn{7}{|l|}{ RMSD } \\
\hline Bond lengths $(\AA)$ & 0.022 & 0.020 & 0.020 & 0.021 & 0.021 & 0.022 \\
\hline Bond angles $\left({ }^{\circ}\right)$ & 1.649 & 1.653 & 1.635 & 1.618 & 1.596 & 1.638 \\
\hline \multicolumn{7}{|c|}{$\begin{array}{l}\text { Experimental procedures are described in the Supplementary Data. } \\
\text { a Values for the highest resolution shell are in parentheses. }\end{array}$} \\
\hline \multicolumn{7}{|c|}{${ }^{\mathbf{b}} R_{\text {symm }}=\sum_{j}\left(I_{j}-\bar{I}_{j}\right) / \sum_{j} I_{j}$ where $\bar{I}_{j}$ is the averaged intensity for symmetry-related reflections. } \\
\hline${ }^{\mathrm{c}} R_{\mathrm{work}}=\sum_{h k l}\left|F_{o}-F_{c}\right|$ & $/ \sum_{h k l} F_{o} 5 \%$ of th & data that were $\mathrm{e}$ & xcluded from the & refinement were 1 & lcula & \\
\hline
\end{tabular}

the phosphorothioate substrate tetraethyl dithiopyrophosphate (TEDTPP) and its phosphorothiodiester hydrolytic product diethyl thiophosphate (DETP) (Fig. 1). The hydrolytic activity of OpdA towards TEDTPP was confirmed by liquid chromatography / mass spectrometry, demonstrating quantitative conversion to DETP in less than $12 \mathrm{~h}$ at $4{ }^{\circ} \mathrm{C}$, whereas no sign of hydrolysis was observed in the absence of OpdA. In this instance, although a model of the Michaelis complex was not captured, the bridging coordination mode of DETP could be seen more clearly, unequivocally showing the sulfur atom tightly coordinated to the $\beta$-metal and the new hydroxyl group terminally coordinated to the $\alpha$-metal (Fig. 2f). In contrast, and in a situation analogous to the 450-h EPO soak, after $>500$ h cocrystallization the coordination mode involved displacement of the bridging hydroxide (Fig. 2g). We therefore soaked the crystals in DETP, which resulted in the phosphorothiodiester displacing the $\mu$-hydroxo bridge in an identical manner to the phosphodiester DEP. This series of complexes establishes that (i) phosphotriester and phosphorothionate substrates loosely coordinate to the $\beta$-metal ion, (ii) nucleophilic attack on either class of substrate immedi- ately results in the product bridging the $\alpha$ and $\beta$ metal ions, (iii) rebinding of the anionic phosphodiester or phosphorothiodiester results in a different coordination mode involving displacement of the $\mu$-hydroxo bridge.

\section{The contribution of ground-state destabilization to catalysis}

Recent theoretical ${ }^{18}$ and spectroscopic ${ }^{15}$ data suggest that tight binding of phosphotriesters at the $\beta$-metal results in the $\mu$-hydroxo bridge becoming more closely associated with the $\alpha$-metal, increasing its nucleophilicity by effectively making a pseudoterminal hydroxide. These studies were based on the assumption that the Michaelis complex involves the substrate becoming tightly coordinated to the $\beta$-metal ion in the same manner as inhibitors. We have previously proposed that substrates do not become tightly coordinated to the $\beta$-metal ion based on density functional theory (DFT) calculations with the substrate methyl parathion. ${ }^{19}$ Additionally, computational analysis has indicated substrates are destabilized through desolvation, ${ }^{20}$ whereas tight metal coordination results in stabilization. ${ }^{21}$ The 

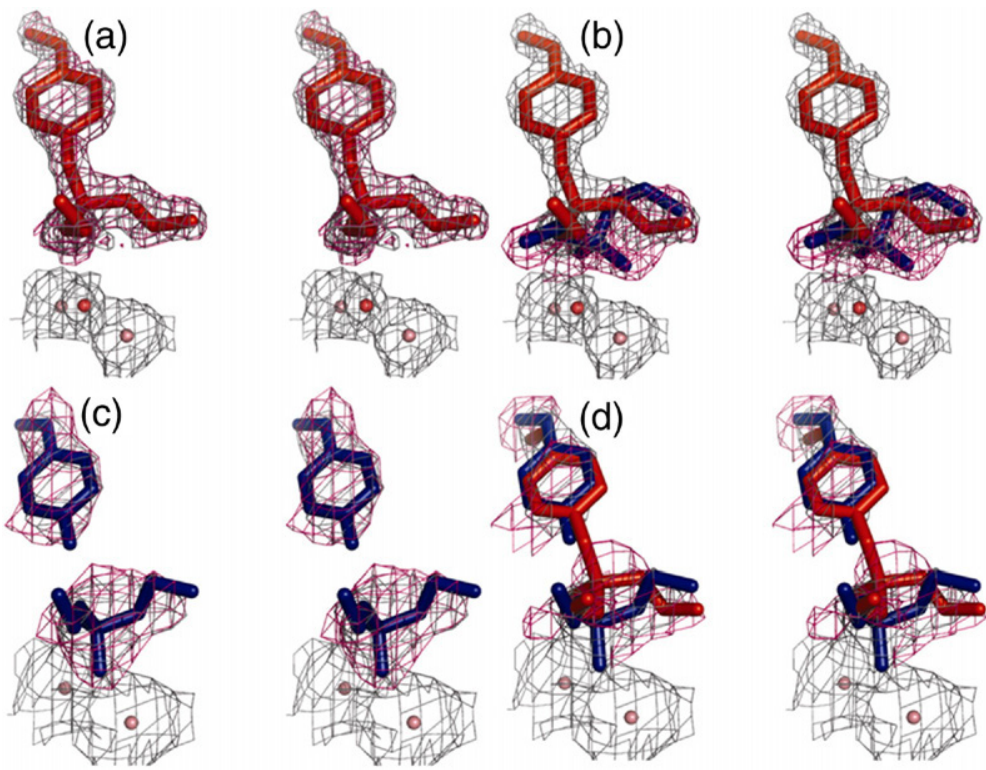

(e)

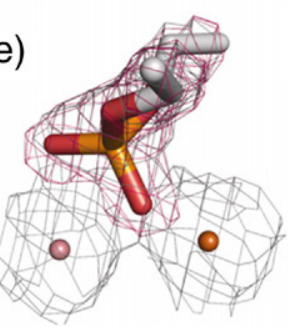

(f)
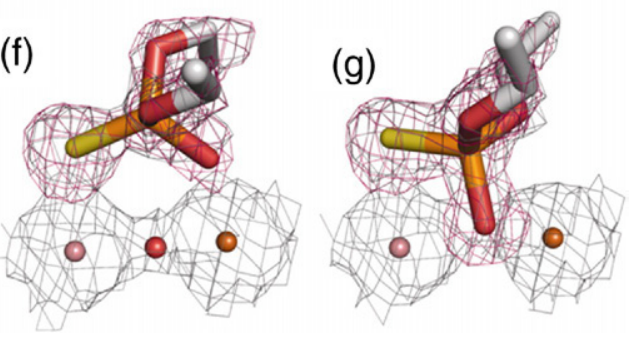

Fig. 2. Substrate and product coordination modes. Omit electron density maps calculated in the absence of bound ligands. $2 \mathrm{~m} F_{\mathrm{o}}-\mathrm{D} F_{\mathrm{c}}$ maps are contoured at $1 \sigma$ (grey). $\mathrm{m} F_{\mathrm{o}}-D F_{\mathrm{c}}$ electron density maps, calculated from models refined in the absence of bound ligands, are contoured at $3 \sigma$ (magenta). For (a) to $(\mathrm{d})$, substrate is coloured red and product is coloured blue. After $20 \mathrm{~h}$, electron density corresponding to EPO was visible in the 1.7- $\AA$-resolution structure (a). Once EPO was included in the model, residual density consistent with DEP bridging the metals became apparent (b). After $450 \mathrm{~h}$, omit electron density indicated substantial breakdown of EPO had occurred in the 2.5- $\AA$-resolution structure. DEP was modelled in a chelating mode, displacing the $\mu$-hydroxo bridge and the leaving group, 4-methoxyphenolate was modelled into the leaving group channel (c). Residual density suggests there was still a small amount of substrate present (d). DEP soaked directly into the crystals displaced the $\mu$-hydroxo bridge (e). TEDTPP was soaked into an OpdA crystal for $20 \mathrm{~h}$, and omit electron density maps show the product DETP bridging the metal ions (f). When TEDTPP was cocrystallized with OpdA (>500 h) or DETP was soaked directly into the crystals, DETP displaced the $\mu$-hydroxo bridge (g).

structures presented here suggest that the withdrawal of the neutral substrate from solvent and binding within the hydrophobic cavity is at least as important as the interaction with the $\beta$-metal ion, and that it is the transition state that coordinates tightly to the positively charged binuclear metal

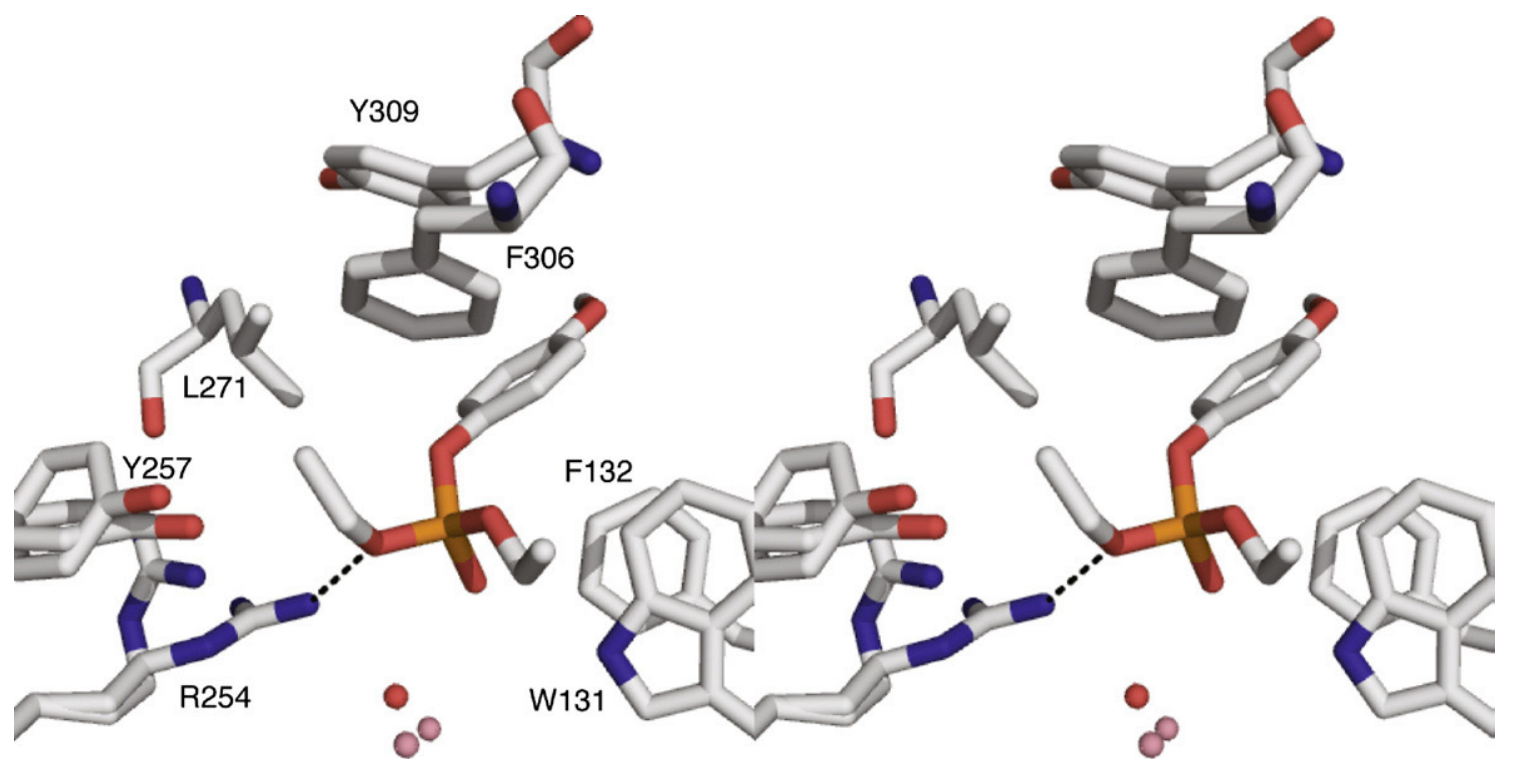

Fig. 3. Conformation of EPO in the active site of OpdA. EPO binds loosely to the $\beta$-metal ion and is hydrogen bonded to R254. Multiple conformations of R254 and Y257 are shown, illustrating their different conformations in the presence and absence of substrate. EPO is significantly stabilized through extensive $\pi-\pi$ interaction between its aromatic ring and the side chains of W131, F132, F306 and Y309. 
centre. This will allow the developing negative charge to be stabilized, thereby lowering the activation energy. Accordingly, catalysis of the hydrolysis of phosphotriesters by the bacterial PTEs appears to result from reduction in the activation energy through a combination of ground-state destabilization and transition-state stabilization.

\section{Comparison between experiment and theory: molecular dynamics docking studies and density functional theory calculations}

Support for a $\mu$-hydroxo bridge nucleophile has been derived from molecular dynamics (MD) simulations that indicated the alignment of the substrate at the active site would be ideal for in-line attack. ${ }^{21}$ These simulations did not include a terminal hydroxide in the model and the substrate orientations obtained are significantly different from the crystallographic complex described here (Fig. 2a). Accordingly, MD-based docking studies were performed using CDOCKER, ${ }^{22}$ in which models did or did not contain a terminal water/hydroxide. This process involves the utilization of a CHARMm ${ }^{23}$ based MD simulation scheme in which random ligand conformations generated from high-temperature MD are translated into the binding site. Binding poses are then searched for using random rigidbody rotations and simulated annealing with a grid potential before minimization with a full force field potential is used to refine the ligand poses. The results are surprising in that the substrate becomes optimally aligned for hydrolysis in both models (Fig. 4). However, the crystallographic structure closely superimposes with the binding mode obtained from the model containing a terminal hydroxide at the $\alpha$-metal. This provides further support for the proposition that a terminally coordinated water/hydroxide molecule is the initiating nucleophile and highlights the importance of the starting model to MD and substrate docking calculations.

Comparison between the distances and alignments of the two putative nucleophiles with the electrophilic phosphorus and the scissile bond in the crystal structure was also undertaken. DFT calculations were used to provide idealised reference structures of reaction intermediates and transition states at the B3-LYP/6-31+G(d) level of theory. Firstly, the distance from the $\mu$-hydroxo bridge to the electrophilic phosphorus of EPO is $3.6 \AA$; in contrast, a terminally coordinated nucleophile would be $3.0 \AA$ from the electrophilic phosphorus, which is close to the $\mathrm{P}-\mathrm{OH}^{-}$distance in the transition state $(2.8 \AA)$. Secondly, the alignment between the $\mu$-hydroxo bridge, electrophilic phosphorus and the leaving group is not ideal for in-line attack. The dihedral angle between the nucleophile, phosphoryl oxygen, phosphorus and leaving group oxygen is significantly different to the idealised alignment, and attack from the $\mu$ hydroxo might be obscured by a side-chain oxygen. In contrast, the alignment of a terminal hydroxide
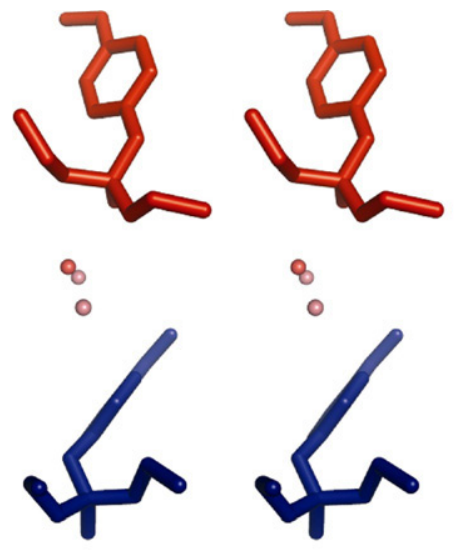

8
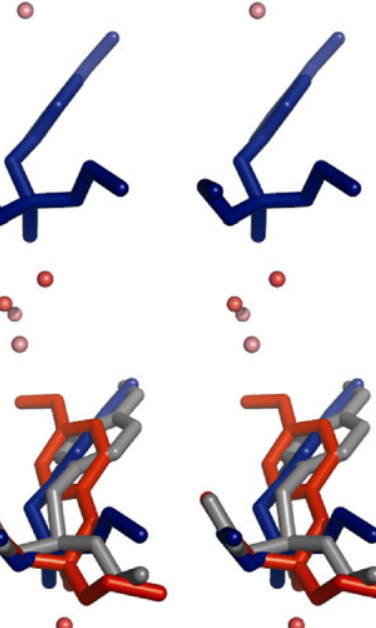

8
8

$\odot$

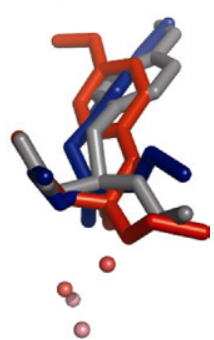

Fig. 4. Comparative MD docking simulations. The most favoured conformations of EPO in the active site of OpdA from CDOCKER, as implemented in the Accelrys Discovery Studio. Hydrogen atoms were added to the protein molecule and EPO, default settings were used, and the CHARMm force field was used to assign partial charges to the ligands. EPO was docked into OpdA models containing (top, red) only a $\mu$-hydroxo bridge and (middle, blue) a $\mu$-hydroxo bridge and a terminally coordinated hydroxide molecule. Both docking poses are aligned for nucleophilic attack, but the crystallographic structure of EPO in the active site (grey) is very similar to the pose aligned for attack from a terminal water molecule when the three structures are superimposed (bottom).

is almost identical with the idealized alignment (Table 2).

\section{Analysis of the promiscuous phosphodiesterase activity of OpdA reveals a general base function for the $\mu$-hydroxo bridge}

The crystal soaking and cocrystallization experiments shown in Fig. 2 strongly suggest that phosphotriesters coordinate terminally to the $\beta$-metal ion, whereas coordination of phosphodiesters results in the displacement of the $\mu$-hydroxo bridge. A comparison between phosphodiester and phosphotriester hydrolysis could therefore reveal the function of the $\mu$-hydroxo bridge, since it will be absent during phosphodiester hydrolysis. At pH 9.0, the $k_{\text {cat }} / K_{\mathrm{m}}$ for the phosphodiesterase activity was several orders of magnitude lower than that of the PTE activity $\left(1.1 \times 10^{-2}\right.$ versus $\left.1.0 \times 10^{6} \mathrm{~s}^{-1} \mathrm{M}^{-1}\right)$, consistent with the characterization of the phosphodiesterase activity in $\mathrm{OPH} .{ }^{24}$ Having established 
Table 2. Distances between the nucleophile, phosphorus and leaving group, and dihedral angles between the hydroxide, phosphoryl oxygen, phosphorus and leaving group oxygen

\begin{tabular}{lcc}
\hline & $\begin{array}{c}\mathrm{OH}^{-}-\mathrm{P} \\
\text { distance }(\AA)\end{array}$ & $\begin{array}{c}\text { Dihedral angle } \\
\left(\mathrm{OH}^{-}-\mathrm{O}=\mathrm{P}-\mathrm{OAr}\right)\end{array}\left(^{\circ}\right)$ \\
\hline $\begin{array}{l}\text { Idealized } \\
\text { transition state }\end{array}$ & 2.8 & 177.4 \\
$\begin{array}{l}\text { Terminal OH } \\
\mu \text {-Hydroxo bridge }\end{array}$ & 3.0 & 178.2 \\
\hline
\end{tabular}

For comparison between the experimentally determined geometries of the reactants with idealised geometries, the optimized geometries of reactants, products, transition states and intermediates for the $\mathrm{S}_{\mathrm{N}} 2$ substitution reaction between EPO and hydroxide were obtained via DFT calculations using Gaussian $03,{ }^{27}$ at the B3-LYP $/ 6-31+\mathrm{G}(\mathrm{d})$ level of theory. The terminal hydroxide was modelled at $2.5 \AA$ from the $\mu$-hydroxo bridge and $\alpha$-metal ion based on positive electron density.

that reliable kinetic data could be obtained, we performed $\mathrm{pH}$ activity analysis of phosphodiester and phosphotriester hydrolysis by OpdA. The estimated $\mathrm{p} K_{\mathrm{a}}$ values of the nucleophiles in the PTE and phosphodiesterase reactions are 4.2 and 6.1, respectively. The OpdA used in this work contains $\mathrm{Fe}^{2+}$ at the $\alpha$-metal ion site, ${ }^{4}$ which makes the $\mathrm{p} K_{\mathrm{a}}$ value of this species (4.2) consistent with the $\mathrm{p} K_{\mathrm{a}}$ values of the nucleophiles in $\mathrm{Zn}^{2+}-(5.8)$ and $\mathrm{Mn}^{2+}$ - (7.3) substituted OPH, ${ }^{2,15}$ when compared to the $\mathrm{p} K_{\mathrm{a}}$ values of the $\mathrm{M}^{2+}-\mathrm{OH}^{-}$species $(6.7,8.4$ and 10.6, respectively). ${ }^{25}$ Since phosphotriesters bind terminally, without disruption of the $\mu$-hydroxo bridge (Fig. 2a), and anionic substrates bind bidentately, displacing the $\mu$-hydroxo bridge (Fig. 2e), this $\mathrm{pH}$ activity analysis demonstrates that the bacterial PTEs are (i) catalytically active in the absence of a $\mu$-hydroxo bridge and (ii) generate a nucleophile at significantly lower $\mathrm{pH}$ values when a $\mu$-hydroxo bridge is present, consistent with a general base function.

\section{Reconciliation of the catalytic mechanism}

One line of experimental evidence supporting the proposition that the $\mu$-hydroxo bridge is the attacking nucleophile in the reaction was the observation that the $\mathrm{p} K_{\mathrm{a}}$ of the attacking nucleophile in the $\mathrm{Zn}^{2+}$ substituted enzyme is between 5 and $6 .{ }^{8}$ This value is considerably lower than the $p K_{a}$ value of 9.0 that has been reported for $\mathrm{Zn}^{2+}-\mathrm{OH}^{-} \cdot{ }^{25}$ Additionally, the $\mathrm{p} K_{\mathrm{a}}$ of the $\mu$-hydroxide bridge is approximately equal to the $\mathrm{pK}_{\mathrm{a}}$ of the single ionizable group responsible for the increase in the catalytic rate. ${ }^{9}$ The data are difficult to reconcile with the alignment of the Michaelis complex and bridging product coordination modes reported here, as well as previous kinetic results that demonstrated the $\mathrm{p} K_{\mathrm{a}}$ of the attacking nucleophile is determined by the identity of the metal at the $\alpha$ site. $^{8}$ Recent studies of biomimetic complexes that have shown $\mu$-hydroxo bridges are poor nucleophiles in comparison with terminally coordinated hydroxide ions in freely exchangeable positions. ${ }^{12,26}$ More important, these studies have established that the $\mathrm{p} K_{\mathrm{a}}$ of terminally bound water molecules can be reduced significantly if they are hydrogen bonded to a nearby $\mu$-hydroxo bridge. Therefore, the absence of any water molecule terminally coordinated to the $\alpha$-metal ion in the resting enzyme should not be considered supporting evidence for a $\mu$-hydroxide nucleophile, as it is an ideal site for a rapidly exchangeable nucleophile. The structures presented here demonstrate that the $\mu$-hydroxide is ideally situated to act as a general base with an incoming water molecule at the terminal position of the $\alpha$-metal ion. This is further supported by the kinetic data illustrating the lower $\mathrm{p} K_{\mathrm{a}}$ value of the nucleophile during catalysis of phosphotriesters, relative to phosphodiesters. Accounting for the general base function of the $\mu$ hydroxo bridge reconciles the previously incompatible catalytic models allowing the $\alpha$-metal ion to influence the $\mathrm{p} K_{\mathrm{a}}$ of the attacking nucleophile, ${ }^{8}$ the

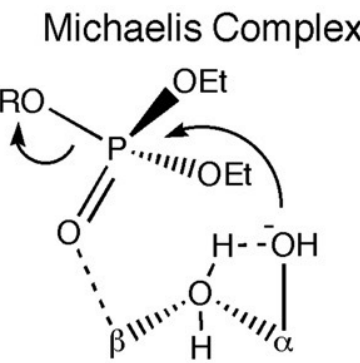

Transition State

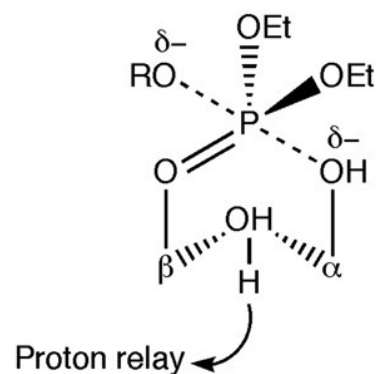

Product<smiles>CCO[P@]12CCO[C@@H]([B]O1)O2</smiles>

Fig. 5. Reaction scheme. In the Michaelis complex, the uncharged substrate is destabilized through desolvation and loosely coordinated to the $\beta$-metal ion. The $\mathrm{p} K_{\mathrm{a}}$ of the water molecule terminally bound to the $\alpha$-metal ion is lowered though its interaction with the $\alpha$-metal ion and the $\mu$-hydroxo bridge, generating a hydroxide nucleophile that is ideally aligned to attack the electrophilic phosphorus. After nucleophilic attack, the developing negative charge on the transition state is stabilized through closer interaction with the positively charged binuclear metal centre, lowering the activation energy. The proton from the water molecule is shuttled from the active site via the $\mu$-hydroxo bridge and the proton relay system. After departure of the leaving group, the phosphodiester bridges the metal ions and the $\mu$-hydroxo bridge remains intact throughout. 
$\mathrm{p} K_{\mathrm{a}}$ of the nucleophile to be similar to the $\mathrm{p} K_{\mathrm{a}}$ of the $\mu$-hydroxide bridge, ${ }^{9}$ only one ionizable group (i.e., the $\mathrm{O}_{2} \mathrm{H}_{3}$ bridge) to be responsible for the increase in the catalytic rate, ${ }^{10}$ and hydrolysis to result in a bridging coordination mode for the product. ${ }^{7}$ Additionally, this model is consistent with the previously proposed proton relay system: ${ }^{8}$ because the $\mu$-hydroxide bridge will become protonated after its participation in the $\mathrm{O}_{2} \mathrm{H}_{3}$ bridge, the proton will need to be shuttled out of the active site. The proposed mechanism is shown schematically in Fig. 5.

\section{Protein Data Bank accession codes}

Structures obtained in this study have been deposited in the Protein Data Bank under accession codes 2R1N, 2R1M, 2R1P, 2R1O, 2R1L and 2R1K.

\section{Acknowledgements}

This work was funded by a grant from the Australian Research Council. We thank the Australian Partnership for Advanced Computing for generous allocations of computer time. We thank the SSRL beam line staff for help with data collection.

\section{Supplementary Data}

Supplementary data associated with this article can be found, in the online version, at doi:10.1016/ i.jmb.2007.10.061

\section{References}

1. Horne, I., Sutherland, T. D., Harcourt, R. L., Russell, R. J. \& Oakeshott, J. G. (2002). Identification of an opd (organophosphate degradation) gene in an Agrobacterium isolate. Appl. Environ. Microbiol. 68, 3371-3376.

2. Serdar, C., Gibson, D., Munnecke, D. \& Lancaster, J. (1982). Plasmid involvement in parathion hydrolysis by Pseudomonas diminuta. Appl. Environ. Microbiol. 44, 246-249.

3. Yang, H., Carr, P. D., McLoughlin, S. Y., Liu, J. W., Horne, I., Qiu, X. et al. (2003). Evolution of an organophosphate-degrading enzyme: a comparison of natural and directed evolution. Protein Eng. 16, 135-145.

4. Jackson, C. J., Carr, P. D., Kim, H.-K., Liu, J.-W., Herrald, P., Mitic, N. et al. (2006). Anomalous scattering analysis of Agrobacterium radiobacter phosphotriesterase: the prominent role of iron in the heterobinuclear active site. Biochem. J. 397, $501-508$

5. Omburo, G. A., Kuo, J. M., Mullins, L. S. \& Raushel, F. M. (1992). Characterization of the zinc binding site of bacterial phosphotriesterase. J. Biol. Chem. 267, 13278-13283.
6. Lewis, V. E., Donarski, W. J., Wild, J. R. \& Raushel, F. M. (1988). Mechanism and stereochemical course at phosphorus of the reaction catalyzed by a bacterial phosphotriesterase. Biochemistry, 27, 1591-1597.

7. Jackson, C., Kim, H.-K., Carr Paul, D., Liu, J.-W. \& Ollis David, L. (2005). The structure of an enzymeproduct complex reveals the critical role of a terminal hydroxide nucleophile in the bacterial phosphotriesterase mechanism. Biochim. Biophys. Acta, 1752, $56-64$.

8. Aubert, S. D., Li, Y. \& Raushel, F. M. (2004). Mechanism for the hydrolysis of organophosphates by the bacterial phosphotriesterase. Biochemistry, 43, 5707-5715.

9. Samples, C. R., Howard, T., Raushel, F. M. \& DeRose, V. J. (2005). Protonation of the binuclear metal center within the active site of phosphotriesterase. Biochemistry, 44, 11005-11013.

10. Dumas, D. P. \& Raushel, F. M. (1990). Chemical and kinetic evidence for an essential histidine in the phosphotriesterase from Pseudomonas diminuta. J. Biol. Chem. 265, 21498-21503.

11. Weston, J. (2005). Mode of action of bi- and trinuclear zinc hydrolases and their synthetic analogues. Chem. Rev. 105, 2151-2174.

12. Kaminskaia, N. V., He, C. \& Lippard, S. J. (2000). Reactivity of mu-hydroxodizinc(II) centers in enzymatic catalysis through model studies. Inorg. Chem. 39, 3365-3373.

13. Carlsson, H., Haukka, M. \& Nordlander, E. (2004). Structural and functional models of the active site of zinc phosphotriesterase. Inorg. Chem. 43, 5681-5687.

14. Hong, S. B. \& Raushel, F. M. (1996). Metal-substrate interactions facilitate the catalytic activity of the bacterial phosphotriesterase. Biochemistry, 35, 10904-10912.

15. Samples, C. R., Raushel, F. M. \& DeRose, V. J. (2007). Activation of the binuclear metal center through formation of phosphotriesterase-inhibitor complexes. Biochemistry, 46, 3435-3442.

16. Beese, L. S. \& Steitz, T. A. (1991). Structural basis for the $3^{\prime}-5^{\prime}$ exonuclease activity of Escherichia coli DNA polymerase I: a two metal ion mechanism. EMBO J. 10, 25-33.

17. Hamdan, S., Carr, P. D., Brown, S. E., Ollis, D. L. \& Dixon, N. E. (2002). Structural basis for proofreading during replication of the Escherichia coli chromosome. Structure, 10, 535-546.

18. Chen, S. L., Fang, W. H. \& Himo, F. (2007). Theoretical study of the phosphotriesterase reaction mechanism. J. Phys. Chem. B, 111, 1253-1255.

19. Jackson, C. J., Liu, J.-W., Coote, M. L. \& Ollis, D. L. (2005). The effects of substrate orientation on the mechanism of a phosphotriesterase. Org. Biomol. Chem. 3, 4343-4350.

20. Zheng, F., Zhan, C.-G. \& Ornstein, R. L. (2001). Theoretical studies of reaction pathways and energy barriers for alkaline hydrolysis of phosphotriesterase substrates paraoxon and related toxic phosphofluoridate nerve agents. J. Chem. Soc., Perkin Trans. 2, 2355-2363.

21. Koca, J., Zhan, C.-G., Rittenhouse, R. C. \& Ornstein, R. L. (2001). Mobility of the active site bound paraoxon and sarin in zinc-phosphotriesterase by molecular dynamics simulation and quantum chemical calculation. J. Am. Chem. Soc. 123, 817-826.

22. Wu, G., Robertson Daniel, H., Brooks Charles, L., 3rd \& Vieth, M. (2003). Detailed analysis of grid-based molecular docking: a case study of CDOCKER-A 
CHARMm-based MD docking algorithm. J. Comput. Chem. 24, 1549-1562.

23. Brooks, B. R., Bruccoleri, R. E., Olafson, B. D., States, D. J., Swaminathan, S. \& Karplus, M. (1983). A program for macromolecular energy, minimization, and dynamics calculations. J. Comput. Chem. 4, 187-217.

24. Shim, H., Hong, S. B. \& Raushel, F. M. (1998). Hydrolysis of phosphodiesters through transformation of the bacterial phosphotriesterase. J. Biol. Chem. 273, 17445-17450.
25. Perrin, D. D. \& Dempsey, B. (1974). Buffers for $p H$ and metal ion control. Chapman and Hall, London, UK.

26. Bauer-Siebenlist, B., Meyer, F., Farkas, E., Vidovic, D., Cuesta-Seijo, J. A., Herbst-Irmer, R. \& Pritzkow, H. (2004). Correlation of structure and function in oligonuclear zinc(II) model phosphatases. Inorg. Chem. 43, 4189-4202.

27. Frisch, M. J., W.T., G., Schlegel, H. B., Scuseria, G. E., Robb, M. A., Cheeseman, J. R. et al. (2004). Gaussian 03, Revision C.02. Gaussian, Inc., Wallingford, CT. 